\title{
Comparative analysis of construction materials used for fastening elements of six-point safety belts in rally cars
}

\section{ARTICLE INFO}

Received: 29 December 2021

Revised: 21 January 2021

Accepted: 14 February 2021

Available online: 5 March 2022
Safety belts are one of the most significant elements of car equipment classified as passive safety. This paper provides a comparative material analysis of critical components of a trusted manufacturer's six-point harness used in motorsports racing with commercially available imitation belts. Despite the FIA certification labels, the imitation belts are characterized by extremely poor quality in the selection of materials for components such as the snap hooks and the locking sleeves in the central fastening mechanism, posing a real hazard to unaware belt users.

Key words: six-point safety belt, failure, motorsport, passive safety

This is an open access article under the CC BY license (http://creativecommons.org/licenses/BY/4.0/)

\section{Introduction}

Seat belts are one of the most crucial components of passive safety [26]. As a critical feature of the six-point belts used in high-performance sports cars, it is essential to ensure the safety of the driver and/or passenger under high acceleration and overload conditions. The sets of three pairs of belts - shoulder, lap and crotch belts - are equipped on one side with buckles fastened in a central locking mechanism and on the other side with a snap hook, which is connected to the body of the vehicle at the mounting points [1]. Sports seat belts must meet rigorous standards, including the FIA 8853-2016 standard set by the International Automobile Federation [7]. At the same time, FIA homologation for belts is valid for five years from the year of manufacture, after which time belts cannot be used in professional motorsport. Unfortunately on the market appeared a number of substitutes and replicas of belts fully imitating certified six-point safety belts with sewn-on labels that confirm compliance with the FIA standard and specify the specific year of the end of the homologation.

Sport seat belts have two main purposes. The first is increased safety. Due to their design, they provide better protection during an accident than civilian belts. This is associated with the greater number of belts and the fact that sports belts are tightened firmly on the human body without any slack. This keeps the body properly supported during an accident and reduces the risk of hitting interior components. The second role of the sport six-point harness is to stabilize the body position in the seat while driving. During sports competitions, there are significant overloads, such as during braking and turn taking [14]. For this reason, driving a performance car in regular belts would cause the body to move cumbersomely on the seat, which would be a hazardous condition for efficient maneuvering of the vehicle. The use of sport harnesses eliminates this inconvenience, making car steering more comfortable and precise [17, 25].

The elements that attach the belts to the body of the vehicle, i.e. snap hooks, are one of the most crucial elements in sports seat belts, being responsible for transferring all loads. Snap hooks, buckles, clasps, and centre fastening mechanisms should be made from medium-carbon steels in the normalized or quenched and tempered state, or highstrength micro-alloyed steels. Selection of such materials makes the elements characterized by relatively high strength while maintaining adequate toughness. The use of fine-grained tempered martensite microstructure materials is a well-established practice in the automotive industry for many years $[3,9,15]$.

Seat belt failure cases described in the literature focus on damage of the polymer fibres subject to degradation processes $[10,11]$, disintegration of the fastener mechanisms $[6,8,21]$ or poor quality of the attachment points to the vehicle body [12]. In addition, an extensive part of the available research results describe medical aspects of postaccident injuries and accident statistics related to passive safety $[5,13,19,20,22,23]$. The referenced articles describe cases of failures that result from many different factors. Most of these relate to the degradation processes of the polymers used in fastener components. Long-term functioning in unfavourable environmental conditions (elevated temperature, exposure to ultraviolet radiation, exposure to oxygen) results in gradual deterioration of the material's mechanical properties. In the end, the conclusions of these analyses usually come down to an improvement of the material selection or construction process, which must take into account nonstandard conditions of use. At the same time, a utilitarian feature of these analyses is their potential usefulness in forensic science. At the same time, the analysis presented in the following represents a different type of failure case, i.e., deliberate lowering of the quality of the material in order to increase the potential profits of the company under the pretext of selling replica belts with the FIA standard.

\section{Subject of the study and methodology}

The purpose of this study was a comparative analysis of the materials used for the snap hooks securing six-point sport harness belts and selected central fastening mechanisms components. The tests were conducted on three sets of belts, one set being the original set from a well-known and verified manufacturer, while the other two sets were 
imitations of the same company's product. Each harness tested (original or imitation) had an appropriate label with the information required by FIA Standard 8853-2016 (Fig. 1): (1) the FIA standard number; (2) the manufacturer's name, which may be replaced by a logo; (3) the unique serial number of the harness; (4) the homologation number; (5) the year of the expiry date; (6) (optionally) the information about adaptation to the FRH system.

The morphology of the microstructure in the nitaletched state was observed using light and electron microscopy methods. It was analysed using a Phenom XL scanning electron microscope (Eindhoven, Netherlands) and a Nikon Eclipse MA 200 light metallographic microscope (Tokyo, Japan) equipped with a Nikon DS-Fi5 CCD camera. SEM observations were conducted using material contrast (BSE detector, back-scattered electrons) under $15 \mathrm{kV}$ accelerating voltage conditions. Additional microscopic observations were made in the picral-etched state, which reveals the distribution and morphology of the iron carbides without etching the grain boundaries.

The chemical composition of the material of snap hooks was determined by optical emission spectroscopy using a Leco GDS-500A glow discharge analyser (St. Joseph, MI, USA).

Hardness measurements were performed using the Vickers method in accordance with PN-EN ISO 65071:2018-5, taking a series of five or ten measurement points for each measurand (depending on measurement stability) to determine the standard deviation. Measurements with a load of 49.03 N (HV5) and 294.2 N (HV30) were made using a Zwick/Roell ZHV30/zwickiLine hardness tester (Ulm, Germany).
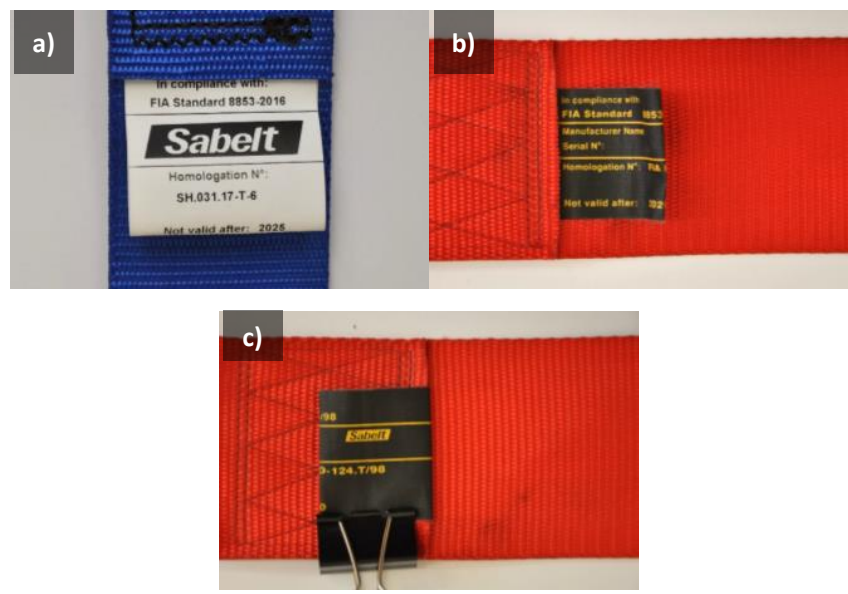

Fig. 1. Manufacturers' labels sewn onto the six-point harnesses containing information regarding compliance with the FIA standard and temporary homologation: a) original belt; b) c) imitations of the original belt

\section{Results and Discussion}

\subsection{Comparative material tests of snap hooks}

It was found that the snap hooks were made of two different grades of steel with various microstructure morphology and hardness. The original component was made of medium-carbon steel of grade C50 according to PN-EN ISO 6831:2018-09. At the same time, it is characterized by the microstructure of tempered martensite, which indicates that the heat treatment process was carried out (Fig. 2a, Fig. 3a).
In case of the snap hook coming from the belt imitating the original one, it was made of low-carbon steel for cold forming of DC04 grade according to PN-EN 10130:2009 standard. It is characterized by a ferritic-pearlitic microstructure with locally occurring precipitations of tertiary cementite at grain boundaries (Fig. 2bc, Fig. 3bc). According to PN-EN 10130:2009, the mechanical properties of such steel are guaranteed for only six months after the product is made at the factory available to the customer, which is due to the aging processes occurring in this type of steel. The presence of tertiary cementite in excessive amounts reduce the toughness of steel, especially if it is located at the ferrite grain boundaries [2, 18]. For this reason, its amount, size and morphology are strictly controlled in the automotive low carbon steels in order to achieve a precipitation strengthening effect instead of a deterioration of the mechanical properties (mainly decrease in toughness) [4, 16, 24, 27].

The significant divergence in the chemical composition (primarily in carbon content) and the form of microstructure translates into the results of hardness measurements $482.1 \pm 2.3 \mathrm{HV} 30$ for the original element and $105.6 \pm 1.0$ HV30 for the snap hook from belts imitating the original ones.

Table 1. Results of spectral analysis of the chemical composition of snap hook from original belts

\begin{tabular}{|c|c|c|c|c|c|}
\hline Object & \multicolumn{5}{|c|}{ Chemical composition [wt.\%] } \\
\hline \multirow{2}{*}{$\begin{array}{c}\text { original } \\
\text { component }\end{array}$} & $\mathrm{C}$ & $\mathrm{Mn}$ & $\mathrm{Si}$ & $\mathrm{P}$ & $\mathrm{S}$ \\
\cline { 2 - 6 } & 0.502 & 0.732 & 0.201 & 0.014 & 0.001 \\
\hline
\end{tabular}

Table 2. Results of spectral analysis of the chemical composition of snap hook from belts imitating the original

\begin{tabular}{|c|c|c|c|c|c|}
\hline Object & \multicolumn{5}{|c|}{ Chemical composition [wt.\%] } \\
\hline \multirow{2}{*}{$\begin{array}{c}\text { imitation } \\
\text { component }\end{array}$} & $\mathrm{C}$ & $\mathrm{Mn}$ & $\mathrm{Si}$ & $\mathrm{P}$ & $\mathrm{S}$ \\
\cline { 2 - 6 } & 0.071 & 0.248 & 0.002 & 0.011 & 0.003 \\
\hline
\end{tabular}

Table 3. Results of HV30 hardness measurements of snap hook from original belts

\begin{tabular}{|c|c|c|c|c|c|}
\hline \multirow{2}{*}{ Object } & \multicolumn{5}{|c|}{ Hardness measurements [HV30] } \\
\hline \multirow{2}{*}{$\begin{array}{c}\text { original } \\
\text { component }\end{array}$} & 482.0 & 478.9 & 480.3 & 479.6 & 481.6 \\
\cline { 2 - 6 } & 486.5 & 483.3 & 484.7 & 482.2 & 481.8 \\
\hline \multicolumn{4}{|c}{ average \pm SD } & $\mathbf{4 8 2 . 1}$ & $\pm \mathbf{2 . 3}$ \\
\hline
\end{tabular}

Table 4. Results of HV30 hardness measurements of snap hook from belts imitating the original

\begin{tabular}{|c|c|c|c|c|c|}
\hline Object & \multicolumn{5}{|c|}{ Hardness measurements [HV30] } \\
\hline \multirow{2}{*}{$\begin{array}{c}\text { imitation } \\
\text { component }\end{array}$} & 103.9 & 105.8 & 103.6 & 105.7 & 106.1 \\
\cline { 2 - 6 } & 106.3 & 106.1 & 106.2 & 105.4 & 106.5 \\
\hline \multicolumn{2}{|c}{ average \pm SD } & $\mathbf{1 0 5 . 6}$ & $\pm \mathbf{1 . 0}$ \\
\cline { 2 - 6 }
\end{tabular}

Conducting a comparative qualitative assessment of the morphology of the two types of microstructures, it is im- 
portant to point out the diametrical differences that are reflected in the contrasting behaviour of the two types of snap hooks:
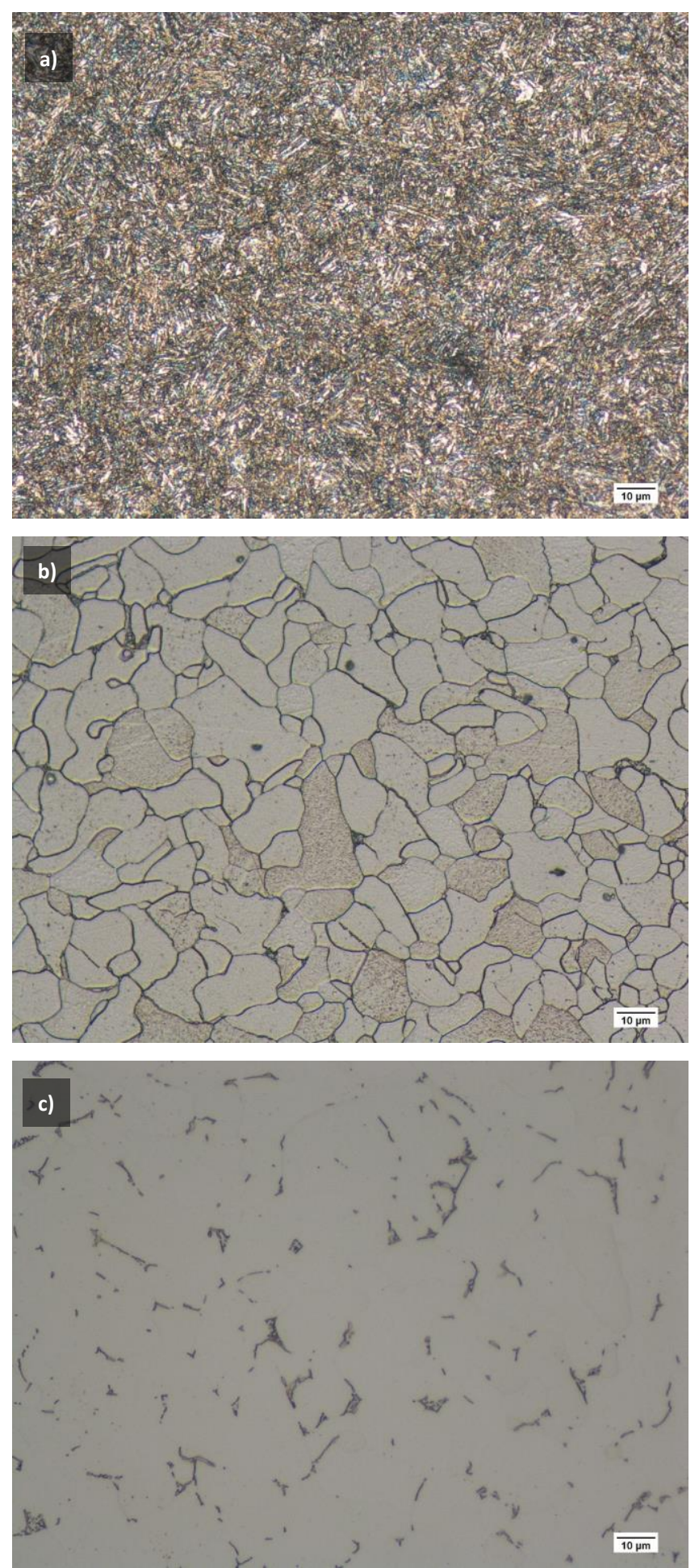

Fig. 2. Snap hook: a) original with tempered martensite microstructure; b) c) imitating original with ferritic microstructure with minor amount of pearlite colonies and presence of tertiary cementite at grain boundaries (micrograph c) in picral-etched state to visualize the extensive amount of cementite)

- the tempered martensite microstructure in the original component in contrast to the ferritic microstructure with a minor amount of pearlite colonies and tertiary cementite precipitations at grain boundaries in the imitation original component translate into the dissimilar material strengthening mechanisms;

- the degree of refinement of the microstructure of the compared elements, which results in a different grain boundary strengthening effect of the material in favour of the original snap hook (Fig. 3a vs Fig. 3c);

- the presence of undesirable precipitations of tertiary cementite at grain boundaries in DC04 steel, which locally forms a continuous network, thus providing a privileged site for accelerated crack growth and propagation;
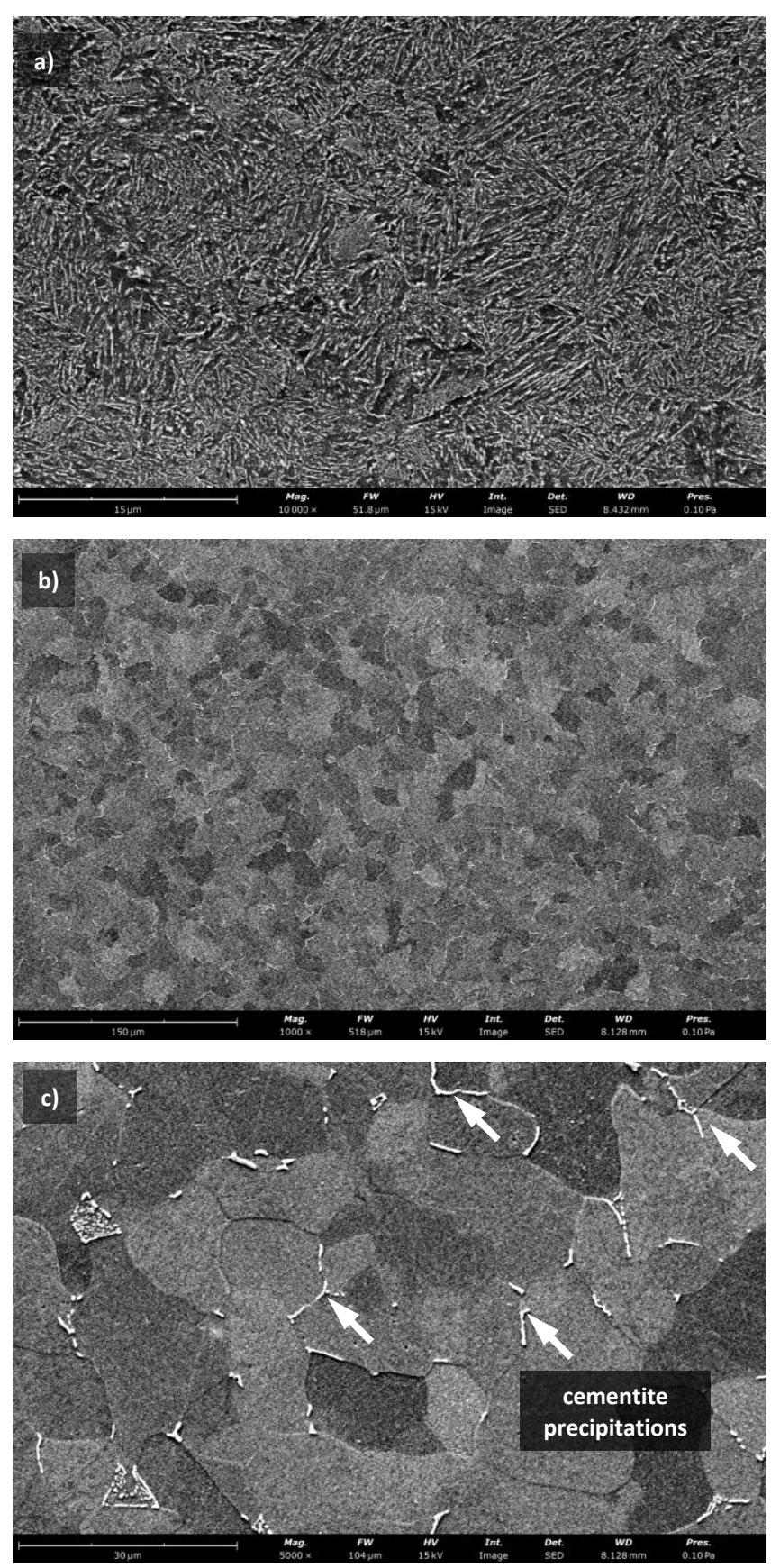

Fig. 3. Snap hook: a) original with tempered martensite microstructure; b) imitating original with ferritic microstructure with minor amount of pearlite colonies; c) numerous tertiary cementite precipitations visible at grain boundaries (indicated by arrows); SEM (BSE) 
- the general purpose of the two identified steels, where C50 in the quenched and tempered condition is intended for highly stressed parts (components in general mechanical engineering and vehicle construction) and DC04 for metal forming (automotive industry, the domestic appliances sector, metal furniture, shaft processing, radiators, ventilators, tubes and small profiles).

According to PN-EN 10130:2009, DC04 steel has a maximum tensile strength of 270-350 MPa. In contrast, C50 grade steel in the quenched and tempered state according to PN-EN ISO 683-1:2018-09 has a tensile strength of 750-900 MPa. These differences are depicted by the almost five times higher hardness of the original snap hook than that of the replica.

\subsection{Comparative material tests of central fastening mechanism components}

A recurring defect of imitation seat belts is the tendency for the central fastening mechanism to jam, making it extremely difficult for the driver and/or passenger to safely and quickly exit the vehicle. The reason for this phenomenon is the deformation of the set of locking sleeves of the seat belt tongues in the central mechanism (Fig. 4). Their size and construction make it impossible to perform chemical composition analysis by GDS methods.
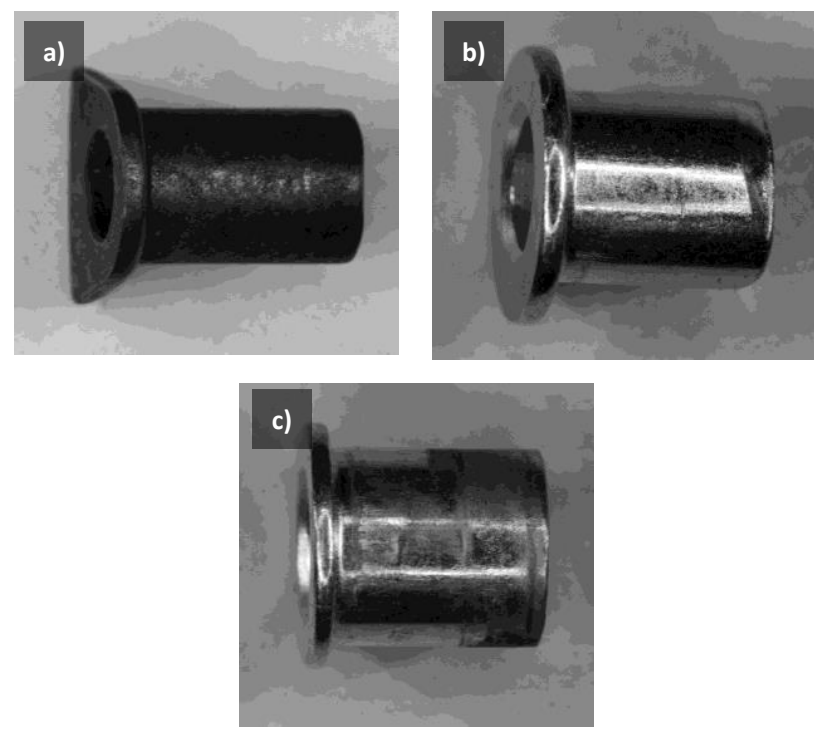

Fig. 4. The central fastening sleeve, which causes the mechanism to jam in the event of an accident, making it difficult to release the safety belts: a) original belts; b) c) imitation of original belts

The original sleeve is characterised by a tempered martensite microstructure with numerous visible porosities and shrinkage microcavities (Fig. 5a). Their presence is an evidence of the casting nature of sleeve production. However, the number and distribution of voids reduce the quality of the material. They provide an easy path for crack propagation. Due to the morphology of the microstructure, the material can be classified as a medium carbon steel for heat treatment.

The sleeves installed in the central fasteners of the belts imitating the original ones are characterised by a microstructure of ferritic-pearlitic morphology with a locally visible deformation texture resulting from the plastic forming pro- cess of the details. This texture translates into anisotropy of the mechanical properties of the sleeve. The number of pearlite colonies is relatively small and their distribution is not band-shaped. The ratio of the proportion of pearlite to ferrite grains makes it possible to classify the material in the family of low-carbon steels for metal forming.
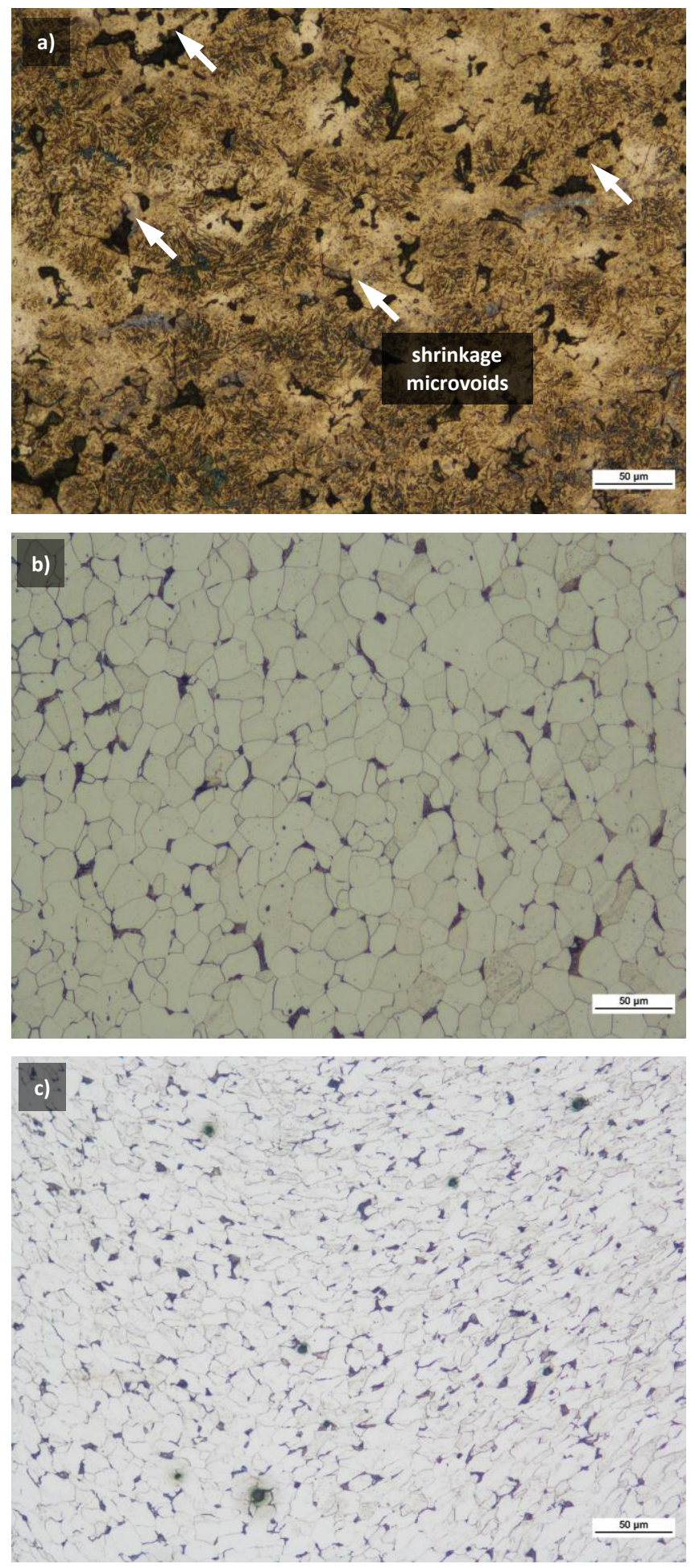

Fig. 5. Sleeve of the central fastener: a) the original with tempered martensite microstructure with visible numerous porosities and shrinkage microcavities; b) c) an imitation of the original with ferritic-pearlitic microstructure with visible locally occurring deformation grain texture resulting from the metal forming process of the workpiece 
The obtained hardness results confirm and illustrate the differences in the materials used for the central fastener elements. In the case of the original part, a hardness of $353.6 \pm 57.8$ HV5 was obtained, while the components imitating the original ones had a hardness of $168.7 \pm 1.8 \mathrm{HV} 5$ and $229.6 \pm 9.4 \mathrm{HV} 5$, respectively. The high standard deviation in the case of the original sleeve is due to the presence of numerous porosities and shrinkage microcavities.

The differences in manufacturing technology (cast steel vs steel), microstructure morphology and design are reflected in the different behaviour of components when subjected to external forces during a potential accident/impact. Due to the use of low-carbon steel, non-original parts are plastically deformed, causing the central fastening mechanism to jam. The original component used is heat-treated and has up to twice the hardness, but the amount of voids and porosity reduces the quality of the material applied, which can also result in cracking and blocking of the central fastening mechanism.

Table 5. Results of HV5 hardness measurements of sleeve from original belts

\begin{tabular}{|c|c|c|c|c|c|}
\hline \multirow{2}{*}{ Object } & \multicolumn{5}{|c|}{ Hardness measurements [HV5] } \\
\hline \multirow{2}{*}{$\begin{array}{c}\text { original } \\
\text { component }\end{array}$} & 305.0 & 298.0 & 326.1 & 450.0 & 352.4 \\
\cline { 2 - 6 } & 439.2 & 410.8 & 313.6 & 311.4 & 329.8 \\
\hline \multicolumn{4}{|c}{ average \pm SD } & $\mathbf{3 5 3 . 6}$ & $\pm \mathbf{5 7 . 8}$ \\
\cline { 2 - 6 }
\end{tabular}

Table 6. Results of HV5 hardness measurements of sleeve A from belts imitating the original

\begin{tabular}{|c|c|c|c|c|c|}
\hline Object & \multicolumn{4}{|c|}{ Hardness measurements [HV5] } \\
\hline $\begin{array}{c}\text { imitation } \\
\text { component } \\
\text { A }\end{array}$ & 171.7 & 168.9 & 167.7 & 168.3 & 166.9 \\
\hline \multicolumn{2}{|c|}{ average \pm SD } & $\mathbf{1 6 8 . 7}$ & $\pm \mathbf{1 . 8}$ \\
\hline
\end{tabular}

Table 7. Results of HV5 hardness measurements of sleeve B from belts imitating the original

\begin{tabular}{|c|c|c|c|c|c|}
\hline Object & \multicolumn{5}{|c|}{ Hardness measurements [hv5] } \\
\hline $\begin{array}{c}\text { imitation } \\
\text { component } \\
\text { B }\end{array}$ & 225.5 & 234.5 & 242.7 & 227.7 & 214.8 \\
\hline \multicolumn{2}{|c|}{ average \pm SD } & $\mathbf{2 2 9 . 6}$ & $\pm \mathbf{9 . 4}$ \\
\hline
\end{tabular}

\section{Conclusions}

On the basis of the test results obtained, it must be stated that the observed differences in materials illustrate the extreme quality in the selection and manufacture of all sets of belts, which is directly related to the safety, health and life of their users. The differences are visible in the applied constructional solutions, selected steel grades, manufacturing technology, microstructure morphology, and achieved material hardness.

For both of the imitation sports belt harness components considered (snap hooks and central fastening mechanism sleeves), the selection of used materials differed significantly from the original belts of the verified manufacturer. It was found that:

- the original snap hook has seven times higher carbon content $(\mathrm{C}: 0.502 \mathrm{wt} \%)$ in relation to the imitation $(\mathrm{C}$ : $0.071 \mathrm{wt} \%$ ), where carbon is the basic element determining the mechanical properties of unalloyed steels;

- the components used are characterized by different microstructure morphology due to the extremely different heat treatment processes performed;

- the different degree of strengthening of the materials is illustrated by the hardness results, where the original component shows higher hardness (snap hook: $482.1 \pm 2.3$ HV30; sleeve: $353.6 \pm 57.8$ HV5) in relation to the imitation (snap hook: $105.6 \pm 1.0 \mathrm{HV} 30$; sleeve A: $168.7 \pm 1.8$ HV5; sleeve B: $229.6 \pm 9.4$ HV5).

The analysed belts imitating the original ones are not able to meet the requirements of the FIA 8853-2016 standard. Unfortunately, at the same time the user of such belts is not able to visually assess their quality, as the belts are equipped with appropriate labels confirming their homologation. Only during the purchase stage can the credibility of the product be evaluated on the basis of the content of the advertisement or confidence in the dealer. At the same time, the results of the research are also intended to raise consumer awareness of the risks associated with the purchase of passive safety equipment from unreliable sources or/and at discounted prices, which entails real threats to life and health. Moreover, they are the base and starting point for the planned further research: verification of the belts in sledge crush tests with a dummy.

\section{Nomenclature}

FIA Fédération Internationale de l'Automobile

FRH frontal head restraint

GDS glow discharge spectrometry
SEM scanning electron microscope

BSE backscattered electron

\section{Bibliography}

[1] ACAR, B.S. Passive prevention systems in automobile safety. International Encyclopedia of Transportation. 2021, 406-414.

https://doi.org/10.1016/B978-0-08-102671-7.10111-3

[2] BENSCOTER, A.O., BRAMFITT, B.L. Metallography and microstructures of low-carbon and coated steels. Metallography and Microstructures - ASM International. 2004, 588607. https://doi.org/10.31399/asm.hb.v09.a0003761

[3] BILLUR, E. Hot formed steels. Automotive Steels. 2017, 387-411. https://doi.org/10.1016/B978-0-08-100638-2.00012-2

[4] BIN, W., ZHENYU, L., XIAOGUANG, Z. et al. Precipitation behavior of nanoscale cementite in hypoeutectoid steels during ultra fast cooling (UFC) and their strengthening effects. Materials Science and Engineering: A. 2013, 575, 189-198. https://doi.org/10.1016/j.msea.2013.02.071 
[5] BOWMAN, E.J., HARRIS, D.A., BROWN, T.H. Scrotal injury following restrained impact: A risk of misnavigation. Injury Extra. 2007, 38(9), 314-316.

https://doi.org/10.1016/j.injury.2006.12.025

[6] DUNN, R.F., MCSWAIN, R.H., MILLS, T. et al. Failure of plastic press release buttons in automobile seat belts. Engineering Failure Analysis. 2005, 12(1), 81-98. https://doi.org/10.1016/j.engfailanal.2004.05.003

[7] FEDERATION INTERNATIONALE DE L'AUTOMOBILE FIA 8853-2016 - Safety Harnesses. 2016.

[8] FREEMAN, M.D. Traffic Injury Investigation. Forensic Epidemiology. 2016, 315-330. https://doi.org/10.1016/B978-0-12-404584-2.00012-4

[9] HALL, J.N., FEKETE, J.R. Steels for auto bodies. Automotive Steels. 2017, 19-45. https://doi.org/10.1016/B978-0-08-100638-2.00002-X

[10] HENSHAW, J.M., WOOD, V., HALL, A.C. Failure of automobile seat belts caused by polymer degradation. Engineering Failure Analysis. 1999, 6(1), 13-25. https://doi.org/10.1016/S1350-6307(98)00026-0

[11] HENSHAW, J.M., WOOD, V., HALL, A.C. Failure of automobile seat belts caused by polymer degradation. Failure Analysis Case Studies II. 2001, 317-329. https://doi.org/10.1016/B978-0-08-043959-4.50033-X

[12] LI, Y., PENG, H., RONG, B. et al. Analysis and Improvement for the failure of seat belt anchor of a car based on simulation and experiment. Proceedings of the FISITA 2012 World Automotive Congress. 2013, 197, 717-731. https://doi.org/10.1007/978-3-642-33805-2_59

[13] LICHTENSTEIN, M.J., BOLTON, A., WADE, G. Body mass as a determinant of seat belt use. The American Journal of the Medical Sciences. 1989, 297(4), 233-237. https://doi.org/10.1097/00000441-198904000-00007

[14] MERKISZ, J., PIELECHA, I., MARKOWSKI, J. Operating parameters of high performance vehicle engines. Combustion Engines. 2007, 131(4), 3-18. https://doi.org/10.19206/CE-117313

[15] DE MOOR, E., SPEER, J.G. Bainitic and quenching and partitioning steels. Automotive Steels. 2017, 289-316. https://doi.org/10.1016/B978-0-08-100638-2.00010-9

[16] NAGATA, M.T., SPEER, J.G., MATLOCK, D.K. Effect of deformation on cementite precipitation during overaging of low-carbon sheet steel. Scripta Materialia. 2001, 44(6), 899903. https://doi.org/10.1016/S1359-6462(00)00696-5
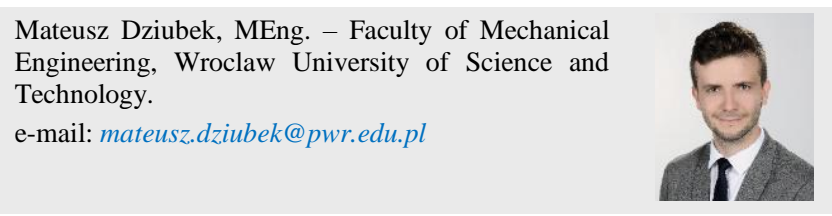

[17] PATALAK, J.P., HARPER, M.G., WEAVER, A.A. et al. Estimated crash injury risk and crash characteristics for motorsport drivers. Accident Analysis \& Prevention. 2020, 136, 105397. https://doi.org/10.1016/j.aap.2019.105397

[18] PERO-SANZ ELORZ, J.A., QUINTANA HERNÁNDEZ, M.J., VERDEJA GONZÁLEZ, L.F. Solid-state transformations in the Fe-C system. Solidification and Solid-State Transformations of Metals and Alloys. 2017, 255-324. https://doi.org/10.1016/B978-0-12-812607-3.00008-5

[19] PORTER, R.S., ZHAO, N. Patterns of injury in belted and unbelted individuals presenting to a trauma center after motor vehicle crash: seat belt syndrome revisited. Annals of Emergency Medicine. 1998, 32(4), 418-424. https://doi.org/10.1016/S0196-0644(98)70169-6

[20] RIVARA, F.P., THOMPSON, D.C., CUMMINGS, P. Effectiveness of primary and secondary enforced seat belt laws. American Journal of Preventive Medicine. 1999, 16(1), 3039. https://doi.org/10.1016/S0749-3797(98)00113-5

[21] ROBERTS, A., PARTAIN, M., BATZER, S. et al. Failure analysis of seat belt buckle inertial release. Engineering Failure Analysis. 2007, 14(6), 1135-1143. https://doi.org/10.1016/j.engfailanal.2006.11.059

[22] SIRIWARDENA, A.K. Seat-belt aortic injury. European Journal of Vascular Surgery. 1990, 4(6), 649-650. https://doi.org/10.1016/S0950-821X(05)80825-4

[23] VAUGHN, M.G., SALAS-WRIGHT, C.P., PIQUERO, A.R. Buckle up: non-seat belt use and antisocial behavior in the United States. Annals of Epidemiology. 2012, 22(12), 825831. https://doi.org/10.1016/j.annepidem.2012.09.010

[24] WANG, B., LIU, Z., ZHOU, X. et al. Precipitation behavior of nanoscale cementite in $0.17 \%$ carbon steel during ultra fast cooling (UFC) and thermomechanical treatment (TMT). Materials Science and Engineering: A. 2013, 588, 167-174. https://doi.org/10.1016/j.msea.2013.09.012

[25] WIDSTROM, L., BLUSTIN, J.M. Motor sports. Sportsrelated fractures, dislocations and trauma. 2020, 901-906. https://doi.org/10.1007/978-3-030-36790-9_63

[26] WRÓBEL, R., SIERZPUTOWSKI, G., HALLER, P. et al. The vehicle driver safety prediction system. Combustion Engines. https://doi.org/10.19206/CE-142950

[27] ZHAO, M.-C., HUANG, X.-F., ATRENS, A. Role of second phase cementite and martensite particles on strength and strain hardening in a plain C-Mn steel. Materials Science and Engineering: A. 2012, 549, 222-227. https://doi.org/10.1016/j.msea.2012.04.040

Dominika Grygier, DSc., DEng. - Faculty of Mechanical Engineering, Wroclaw University of Science and Technology. e-mail:dominika.grygier@pwr.edu.pl 